\title{
Microwave Reflectivity From Gold Sputtered Nanolayer
}

\author{
Pavel Buchar, Student Member, IEEE, Jan Macháč, Senior Member, IEEE, and Ján Zehentner, Fellow, IEEE
}

\begin{abstract}
This paper deals with reflectivity of a gold layer of nanometer-scale thickness sputtered on a dielectric foil at microwave frequencies. A model of nanometer-scale spheres characterizes the behavior of the layer. For short sputtering times, these spheres are separated, and later they touch each other to form a continuous layer. The structure is analyzed in several ways. The first model, as suggested in the literature, replaces the spheres by their electric dipole moments, while the second, newly proposed model replaces them by their mutual capacities. The structure is also being investigated by the CST Microwave Studio software. The data provided by the models is compared with the data obtained from measurements carried out and published by the authors previously. The reduction in effective conductivity of the layer due to microscopic phenomena in thin films is taken into account in the models. The measured conductivity is compared with values taken from the literature, and the differences are discussed.
\end{abstract}

Index Terms-Conductivity, nanoparticle, polarisability, reflection coefficient, thin layer.

\section{INTRODUCTION}

$\mathbf{T}$ HIN metallic layers and nanoparticles have been gaining importance in recent decades, e.g., in the field of so-called active packing [1] and electromagnetic shielding [2]. The behavior of a film of metallic nanometer-scale spheres [3] and rings [4] has been investigated at optical frequencies. The behavior of thin metallic layers at microwave frequencies has been studied in [5], where the authors showed that even a very thin layer can absorb up to $50 \%$ of the energy of an electromagnetic wave. Reference [6] studies the effects of microscopic phenomena occurring in thin layers on their effective conductivity. In [7] there is a study of the electrical conductivity of inhomogeneous thin layers at various frequencies and temperatures.

In [8] and [9] a sputtered gold layer was experimentally investigated at microwave frequencies. It was found that for short sputtering times there is low reflection from a layer of disconnected gold particles, whereas for longer sputtering times there is high reflection from a homogeneous layer tens of nanometers in thickness. In addition, the conductivity of the sputtered layer was measured in [9].

The aim of this work was to find a model that is able to approximate the experimental reflection coefficient data for the

Manuscript received October 3, 2006; revised March 27, 2007. This work was supported by the Grant Agency of the Czech Republic under Project 102/06/ 1106 "Metamaterials, nanostructures and their applications." The review of this paper was arranged by Associate Editor J. O'Brien.

P. Buchar, J. Macháč, and J. Zehentner are with the Czech Technical University, 16627 Praha, Czech Republic (e-mail: bucharp@fel.cvut.cz).

Digital Object Identifier 10.1109/TNANO.2007.907252

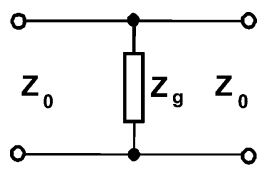

Fig. 1. Equivalent circuit of the layer.

whole range of sputtering times. Our group was involved in investigating gold layers in the past. We therefore dealt only with modeling gold layers. The topology of sputtered layers of other metals differs from those of gold layers. Therefore, the findings presented here are valid only for gold.

Our models are based on replacement of the sputtered layer by a set of gold nanometer-scale spherical particles distributed regularly or randomly on a waveguide aperture. The process of gradual deposition of metal by sputtering is simulated by decreasing the distance between the particles or increasing the number of particles. Interaction of the particles with the electromagnetic waves is described in several ways. The first model [10] replaces the spheres by the induced electric dipole moment. The second, newly proposed model replaces the spheres by their mutual capacities. These two models are valid only for short sputtering times, when the particles are disconnected. For longer sputtering times, a model considering a homogeneous metallic layer is used. Finally, the layer of gold particles is modeled using the CST Microwave Studio software. All resulting curves of the reflection coefficient with respect to sputtering time were compared with measured data.

\section{MODELS OF THE SPUTTERED LAYER}

The models describe only the metallic layer itself, regardless of the polymer foil on which it is sputtered. A metallic layer is replaced by its impedance $Z_{\mathrm{g}}$, which shunts a waveguide with characteristic impedance $Z_{0}$, as sketched in Fig. 1 .

Alternatively, the layer may be described by the scattering matrix with parameters $S_{11}, S_{12}, S_{21}$, and $S_{22}$ bound with the shunt impedance $Z_{g}$ by formulas derived on the basis of circuit analysis, e.g., in [11]

$$
\begin{aligned}
& \mathrm{S}_{11}=\mathrm{S}_{22}=-\frac{1}{1+\frac{2 Z_{g}}{Z_{0}}}, \\
& \mathrm{~S}_{21}=\mathrm{S}_{12}=\frac{1}{\frac{Z_{0}}{2 Z_{g}}+1} .
\end{aligned}
$$

\section{A. Dipole Model}

This model was taken from [10]. It considers a 2-D regular rectangular plane array of electrically small particles placed with distance $a$ illuminated by a perpendicularly incident plane wave $\mathbf{E}_{\text {ext }}, \mathbf{H}_{\text {ext }}$ with wave vector $\mathbf{k}_{\text {ext }}$. This wave excites each 


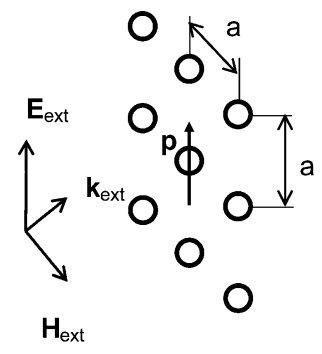

Fig. 2. Array of particles illuminated by an electromagnetic wave.

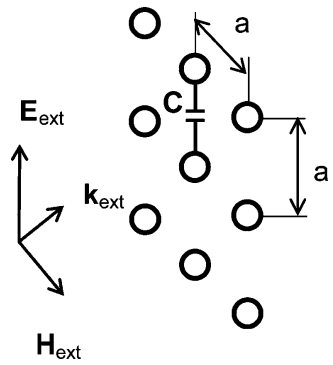

Fig. 3. Idea of mutual capacity between spheres.

particle. Only excitation of dipoles is expected, while multipoles are neglected. The arrangement is sketched in Fig. 2.

The particles are described by polarizability $\alpha$, which links the electrical dipole moment $\mathbf{p}$ with the local electric field $\mathbf{E}_{\mathrm{loc}}$ at the particle location

$$
\mathbf{p}=\alpha \mathbf{E}_{\text {loc }}
$$

$\mathbf{E}_{\mathrm{loc}}$ is the sum of the field of the incident wave and of the fields caused by all other particles. For a sphere, the polarizability is [10]

$$
\alpha=\frac{\varepsilon-\varepsilon_{0}}{\varepsilon+2 \varepsilon_{0}} \varepsilon_{0} 4 \pi r^{3},
$$

where $\varepsilon_{0}$ is the vacuum permittivity, $\varepsilon$ is the complex permittivity of the material forming the sphere; for metal at microwave frequencies $\varepsilon \approx-j \sigma / \omega$ may be assumed. $\sigma$ is the conductivity of the metal and $r$ is the sphere radius. According to [10], the impedance of such an array is

$$
\begin{aligned}
Z_{g}=-j \frac{a^{2}}{\omega}\left[\operatorname{Re}\left(\frac{1}{\alpha}-\beta\right)\right. & \\
& \left.+j\left(\operatorname{Im}\left(\frac{1}{\alpha}\right)-\frac{k_{\mathrm{ext}}^{3}}{6 \pi \varepsilon_{0}}\right)\right] \frac{b_{W}}{a_{W}}
\end{aligned}
$$

where $\omega$ is the angular frequency of the electromagnetic wave, and $\beta$ is the so-called interaction constant

$$
\beta=-j \frac{\omega}{a^{2}} \frac{Z_{0}}{4}\left(1-\frac{1}{j k_{\mathrm{ext}} \frac{a}{1.438}}\right) e^{-j k_{\mathrm{ext}} \frac{a}{1.438}}
$$

$b_{W}$ is the height of the waveguide in which the layer is placed, and $a_{W}$ is its width.

The real part of impedance $Z_{\mathrm{g}}$ represents the absorption losses in the layer, and the imaginary part describes its reactive behavior. This model is valid only for short sputtering times when the spheres are disconnected.

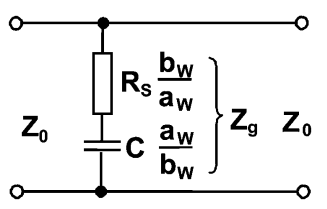

Fig. 4. Equivalent circuit for the combined model.

\section{B. Capacity Model}

The newly proposed model expects the character of the impedance to be capacitive due to the mutual capacity between the spheres, as suggested in Fig. 3.

For simplicity, only the capacity of neighboring spheres is assumed. According to [12], this capacity $C$ is given by the series

$$
\begin{aligned}
C=4 \pi \varepsilon_{0} r^{2}\left(\frac{1}{a}+\frac{r^{2}}{a^{3}-2 a r^{2}}\right. & \\
& \left.+\frac{r^{4}}{a^{5}-4 a^{3} r^{2}+3 a r^{4}}+\ldots\right) .
\end{aligned}
$$

In our calculations we use the first nine terms of this series [12]. For the impedance we get

$$
Z_{g}=\frac{1}{j \omega C} \frac{b_{W}}{a_{W}}
$$

In this case, the value of the impedance is purely imaginary and does not include losses. This model may also be used only for short sputtering times when the spheres are disconnected and their mutual capacity may therefore be assumed.

\section{Thin Layer Model}

Unlike earlier models describing the behavior of individual metallic nanoparticles, this model considers the structure as a continuous layer and is therefore valid only for longer sputtering times. For a layer of thickness $h$, the impedance $Z_{\mathrm{g}}$ is purely real

$$
Z_{g}=\frac{1}{\sigma h} \frac{b_{W}}{a_{W}}=R_{S} \frac{b_{W}}{a_{W}},
$$

where $R_{\mathrm{S}}$ is the square resistivity of the layer.

\section{Combined Model}

The two last-mentioned models may be combined in order to describe both the capacitive behavior of the separate metallic nanoparticles at short sputtering times and the resistive behavior of a homogeneous metallic layer at long sputtering times. The resulting equivalent circuit is shown in Fig. 4. For low sputtering times, when the metallic particles are separated, the impact of capacity is dominant, and the resistivity represents negligible ohmic losses in separated metallic nanoparticles. When the particles touch each other and the layer becomes continuous, the capacity becomes infinity and only the resistivity plays a role.

A similar model was proposed in [7], taking into account the resistivity between the metallic islands, so-called intragranual resistivity, which is connected in parallel with the intragranual capacity, equivalent to our $C$. The authors use the model for calculating the temperature and frequency dependence of the effective resistance of the layer without considering changes with 


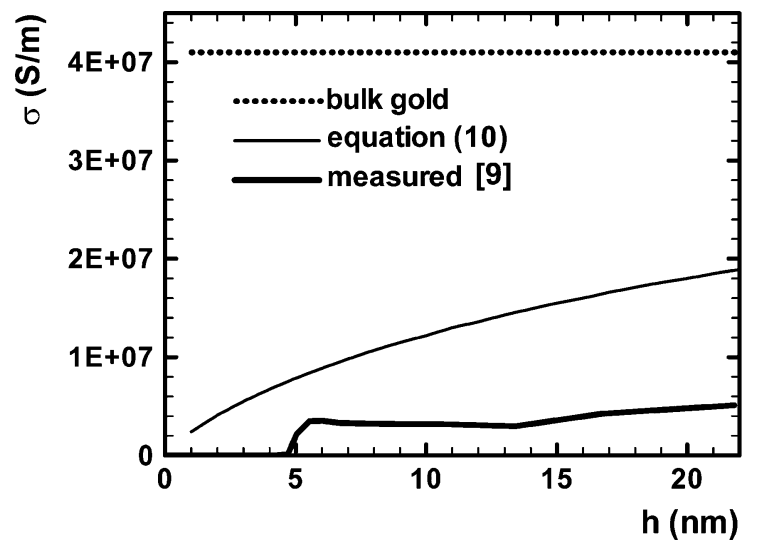

Fig. 5. Layer conductivity against layer thickness.

deposition time. This effective resistance is incomparable with the quantities resulting from our approach.

\section{E. CST Microwave Studio Model}

The layer was also modeled using the CST Microwave Studio [13], which directly provides the scattering parameters. Spheres with conductivity $\sigma$ were either regularly or randomly distributed on a parallel-plate waveguide aperture terminated from the sides by perfect magnetic walls and from the top and the bottom by perfect electric walls.

\section{THIN LAYER CONDUCTIVITY}

It has been shown by many authors that, in the case of thin layers, the commonly used conductivity of a bulk material $\sigma_{0}$ cannot be used. As a result of the microscopic behavior of electrons occurring in thin layers, the conductivity of such a layer is effectively reduced in comparison with $\sigma_{0}$. According to [6], the effective conductivity of a thin layer is

$$
\begin{aligned}
& \sigma=\sigma_{0}\left(1-\frac{3}{8 x}+\frac{e^{-x}}{16 x}\right.\left(6-10 x-x^{2}+x^{3}\right) \\
&\left.+\frac{x}{16}\left(12-x^{2}\right) \int_{x}^{\infty} \frac{e^{-u}}{u} d u\right)
\end{aligned}
$$

where $x=h / \lambda, h$ is layer thickness, and $\lambda$ is the electron mean free path in the material.

In [9], the conductivity of a sputtered gold layer is measured at dc. The measured curve with respect to layer thickness is shown in Fig. 5. The same figure shows the curve obtained by (10) for gold, $\sigma_{0}=4.110^{7} \mathrm{~S} / \mathrm{m}$, and $\lambda=0.057 \mu \mathrm{m}$ [6]. As can be seen, there is an important difference between the two curves. This difference is probably caused by the fact that (10) considers a homogeneous thin layer, while the sputtered layer of metal is porous. In all models we will use the measured conductivity.

\section{COMPARISON OF THEORETICALly COMPUTED AND MEASURED DATA}

The measured sputtering time dependence of the reflection coefficient was taken from [8] and [9]. The measurement set up is depicted in Fig. 6, along with the equivalent circuit of the measured sample and waveguide termination. The gold layers on a polymer foil for various sputtering times were successively
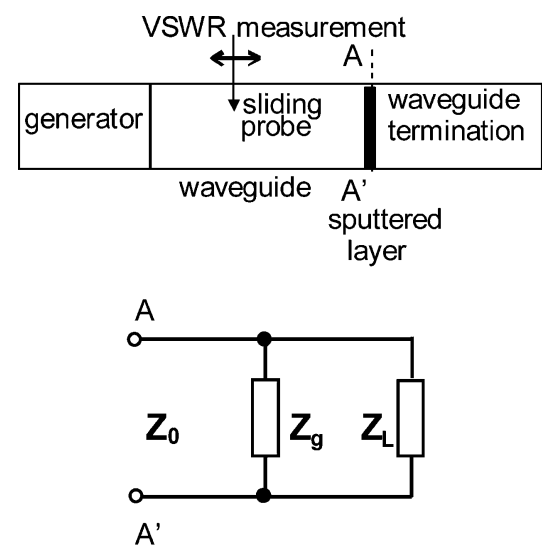

Fig. 6. Measurement setup and its equivalent circuit.

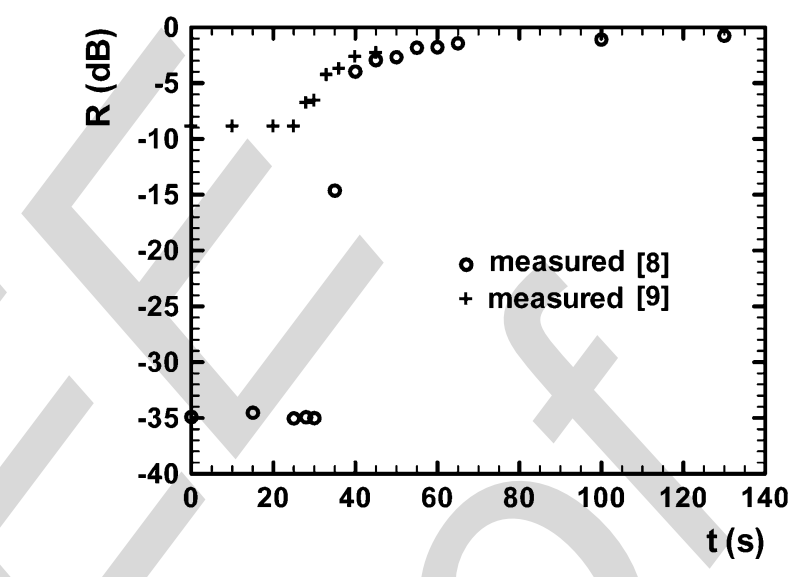

Fig. 7. Measured reflection coefficient against sputtering time according to [8] and [9].

placed in the rectangular WR90 waveguide fed from the left with the mode $\mathrm{TE}_{10}$. A matched load [8], or an open end [9], terminated the waveguide on the right hand side of the layer. In the left part of the waveguide, the voltage standing wave ratio (VSWR) in front of the sample was measured by a slot line. The reflection coefficient was calculated from measured VSWR. The measurement at 8.2 and $12.6 \mathrm{GHz}$ provided results practically identical for both frequencies.

The measured reflection coefficient against sputtering time is shown in Fig. 7. The low reflection corresponds to the nonhomogeneous layer formed by isolated islands of metal. The region of rapid increase corresponds to the mutual touching of the particles and the creation of conducting paths short-circuiting the waveguide. Although the layers in [8] and [9] were prepared under more or less the same conditions, it may be seen that there is a difference of several seconds between the times when the rapid increase in reflection occurs. Apparently, this is caused by the fact that the sputtering and the creation of the conducting path is a random process and, thus, the rapid increase in reflectivity may occur at different times.

The measurement of layer thickness $h$ on particular sputtering time $t$ was performed using the Atomic Absorption Spectroscopy method. Resulting effective $h$ was approximated by a linear formula [8], [9]

$$
h(t)=1.676810^{-10} t,
$$




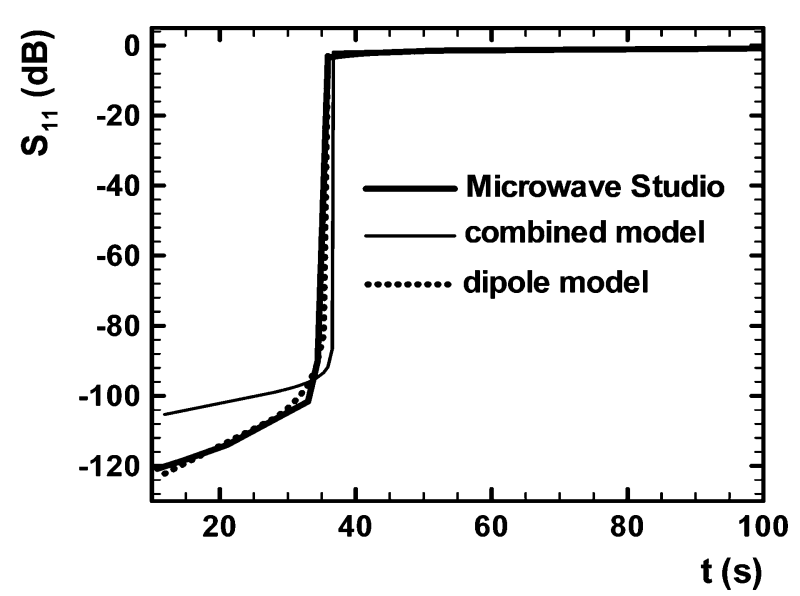

(a)

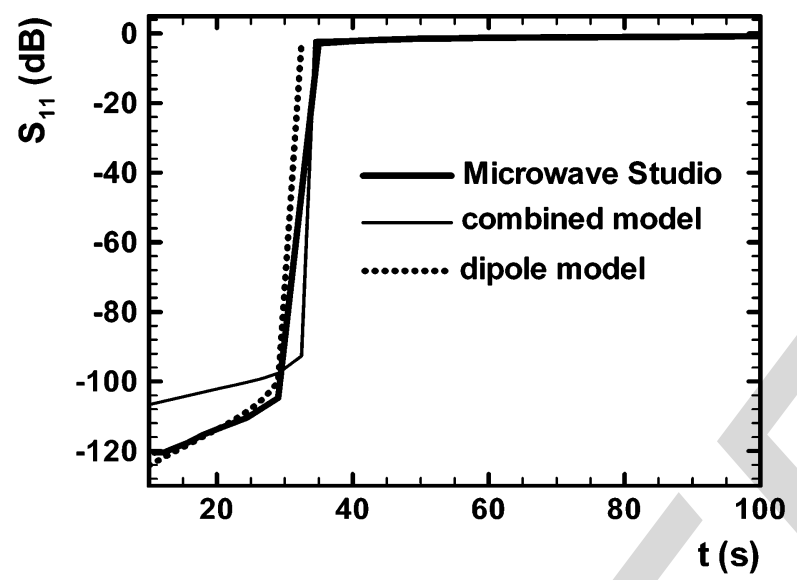

(b)

Fig. 8. $S_{11}$ of the sputtered layer against sputtering time obtained by the different models for: (a) $r=5.75 \mathrm{~nm}$ and (b) $r=5.2 \mathrm{~nm}$ (b).

where $h$ is inserted in meters, and $t$ in seconds.

In order to compare the data obtained from the individual models and from measurement, it is necessary to take into account the impact of the polymer foil and the waveguide termination. This impact may be included into the common reflection coefficient $\Gamma$ of the polymer foil and the waveguide termination. Its value is known from measurement for zero sputtering time and equals $-34.9 \mathrm{~dB}$ for the measurement in [8] and $-10 \mathrm{~dB}$ for the measurement in [9]. In the equivalent circuit, Fig. 6, this impact describes the impedance $Z_{\mathrm{L}}$ related to $\Gamma$ by

$$
\Gamma=\frac{Z_{L}-Z_{0}}{Z_{L}+Z_{0}} .
$$

The scattering parameters from the individual models (1), (2) can be now recalculated to the reflection coefficient of the whole measurement arrangement according to [11]

$$
R=\mathrm{S}_{11}+\frac{\mathrm{S}_{21} \mathrm{~S}_{12} \Gamma}{1-\mathrm{S}_{22} \Gamma} .
$$

In addition, in order to compare the data obtained from individual models with measured data, we have to determine the sputtering time corresponding to the particular distribution of the spheres. In the first step, the layer thickness is computed by dividing the volume of spheres $V$ by the surface of the aperture $S$, on which the spheres are placed

$$
h=\frac{V}{S} .
$$

Subsequently, (11) determines the corresponding sputtering time $t$

Value $h$ is the effective thickness of the layer. It is obtained by the Atomic Absorption Spectroscopy method or by (14) as the average thickness of the nonhomogeneous layer consisting of individual metallic nanoparticles. Since spheres of constant radius are used, this approach is valid only to thickness $h$ equal to the sphere diameter. This state corresponds to a very dense array of spheres, or, in other words, an already homogeneous layer. For higher thicknesses, the concept of spheres is no longer applicable, and the thin layer model considering a homogeneous layer is used.

Although the reflection coefficient was measured in the rectangular waveguide with $\mathrm{TE}_{10}$ mode, and the reflection coefficient determined by particular models is valid for the TEM wave, they can be compared, as we verified by simulations in the CST Microwave Studio.

\section{A. Regular Distribution of Spheres}

At first a regular array of gold spheres with constant radius $r$ was considered. The change in their distance $a$ simulates the gradual sputtering process, during which the number of particles increases and, thus, their distance decreases. This process is applied within the frame of all models. The aperture comprising $8 \times 4$ spheres is used in the CST Microwave Studio model.

The values of $r$ and $a$ unambiguously determine the corresponding value of sputtering time $t$ obtained via (14) and (11); assuming the volume of spheres $V=n(4 / 3) \pi r^{3}$ and the aperture equal to the square of the distance of the spheres multiplied by their number $n$ on the aperture $S=n a^{2}$, we get

$$
t=\frac{\pi r^{3}}{1.6768 \cdot 10^{-10} a^{2}}
$$

where $t$ is inserted in seconds and $r$ and $a$ are in meters. The value of the sphere radius $r$ corresponds to physical size of one piece of the metal gradually forming the sputtered layer. We do not know its value from our macroscopic investigation. However, we can choose it retroactively in such a way that the resulting reflection coefficient fits measured data well.

In order to approximate the measured data from [8] sphere radius $r=5.75 \mathrm{~nm}$ was used, and to approximate data from [9], sphere radius $r=5.2 \mathrm{~nm}$ was used. The calculated time dependences of coefficient $S_{11}$, representing the properties of the layer itself, are shown in Fig. 8.

It is seen that for low sputtering times the reflection is very low, in the order of $-100-120 \mathrm{~dB}$, and it grows slightly until the time of the rapid increase. The time dependences of the resulting reflection coefficient $R$ according to (13), obtained by the particular models and by measurement, are compared with measured data in Fig. 9.

The dipole model is valid only up to the time corresponding to the rapid increase region. The other models are valid within the whole time interval. The curves of the reflection coefficient $R$ fit 


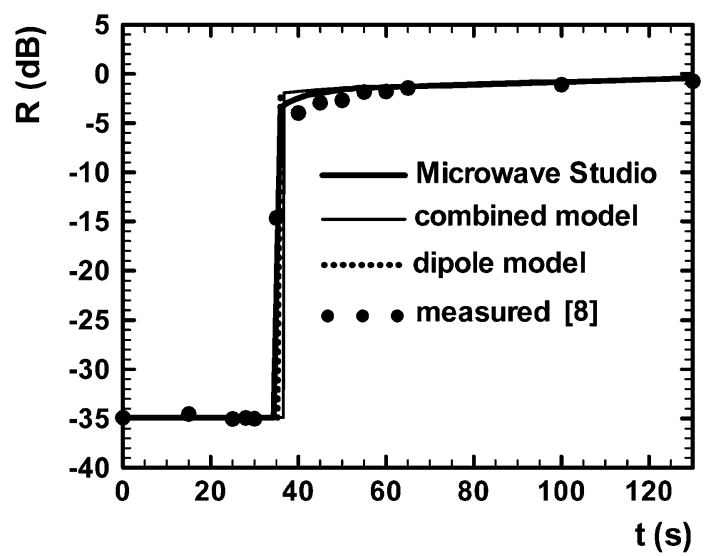

(a)

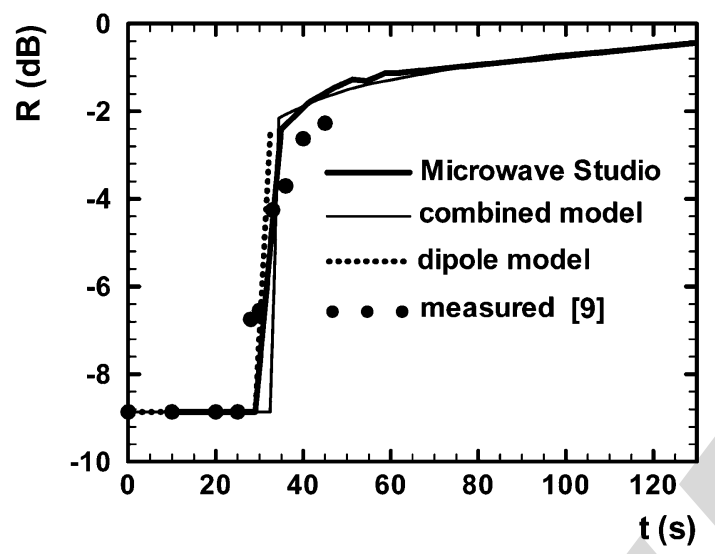

(b)

Fig. 9. Reflection coefficient against sputtering time obtained by the different models for: (a) $r=5.75 \mathrm{~nm}$ and (b) $r=5.2 \mathrm{~nm}$, and compared with the measured data according to (a) [8] and (b) [9].

the measured data well. In addition, it is seen that for low sputtering times the values of reflection coefficient are mainly determined by the reflection from the waveguide termination. The use of two different values of $r$ is in accordance with the abovementioned assumption that sputtering is a random process, despite the more or less identical conditions under which it is performed. It may be assumed that the average dimension of the pieces of material forming the layer differ for each prepared layer.

\section{B. Random Distribution of Spheres}

Consideration of the random distribution of spheres was motivated by the random character of the real sputtering process. The random distribution of the spheres can be taken into account by the CST Microwave Studio model. The positions of the spheres are generated by an internal random generator of uniformly distributed random numbers.

The precedent model taking into account the regular distribution of the spheres has only one degree of freedom determining the final shape of the reflection coefficient curve-sphere radius $r$. The variable simulating the sputtering process in that case is the distance of the spheres $a$. By contrast, the model considering the random distribution of the spheres has two degrees of freedom - sphere radius $r$ and random distribution, because, clearly, for a different random distribution of the spheres the conducting path is created by a different number of spheres and

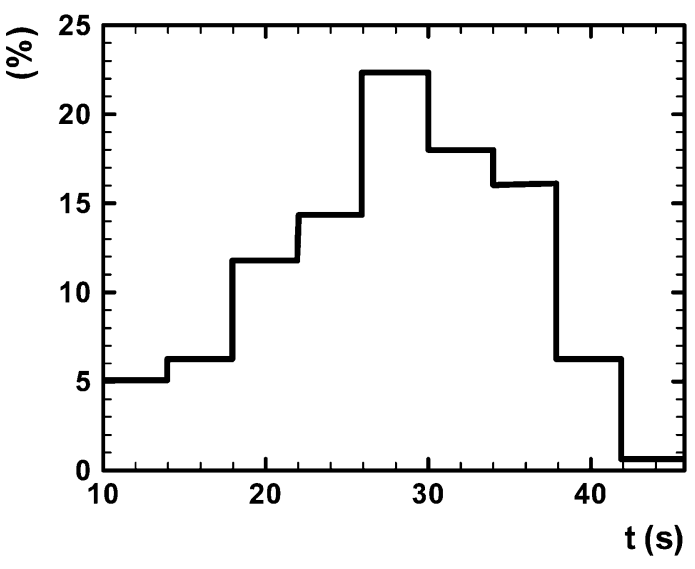

Fig. 10. Probability density of the time of the rapid increase in reflectivity.

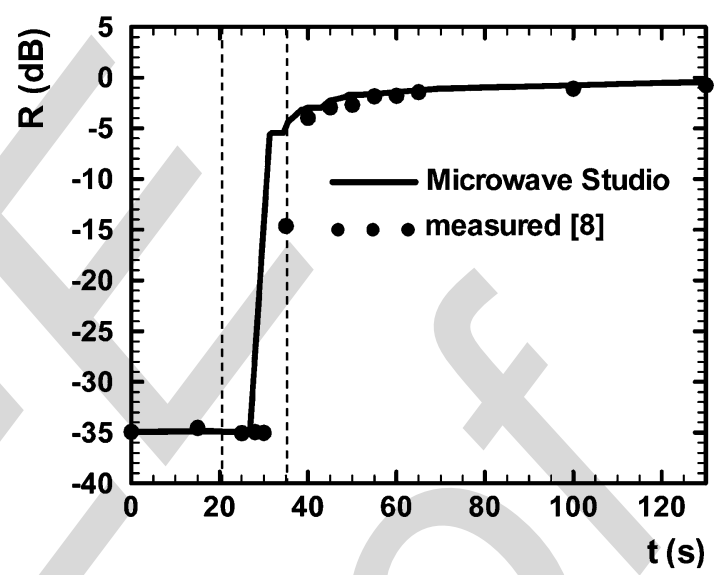

(a)

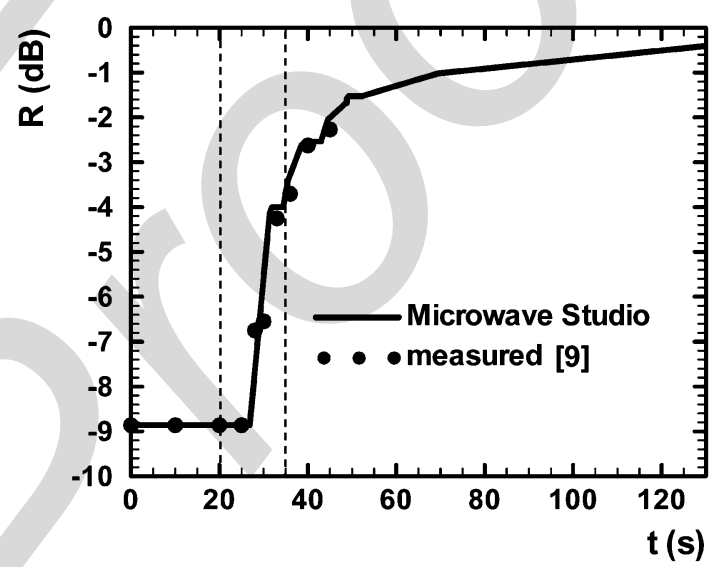

(b)

Fig. 11. The most probable curve of the reflection coefficient against sputtering time compared with measured data [8] (a) and [9] (b). The dashed lines define the interval given by the mean value \pm the standard deviation.

at different corresponding sputtering times and, indeed, the resulting calculated curves of the reflection coefficient also differ. The variable simulating the sputtering process in this case is the gradually growing number of spheres $n$.

For simplicity, we used only one degree of freedom in the CST Microwave Studio simulations, i.e., various random distributions of the spheres, while the sphere radius $r=5.75 \mathrm{~nm}$ remains constant.

In the CST Microwave Studio model, the spheres were randomly distributed on the small part of the waveguide aperture 
with area $S=92 \times 46 \mathrm{~nm}^{2}$. This value was chosen so that the area is sufficiently large to ensure enough spheres ensuring random character of their distribution and, at the same time, a small enough value to make the simulation feasible.

For various simulated random distributions of the spheres, the sputtering time corresponding to rapid increase in reflectivity differs. On the basis of simulations of 162 random distributions, the probability distribution of this time was determined. The mean value of this time is approx. $27.81 \mathrm{~s}$, and the standard deviation is $7.33 \mathrm{~s}$ and $67 \%$ of the times of rapid increase fall in the interval given by the mean value \pm the standard deviation. The corresponding probability density chart is shown in Fig. 10.

The most probable curve of the reflection coefficient is shown in Fig. 11, in comparison with measured data from [8] and [9].

\section{CONCLUSION}

A new approach to sputtered gold layer modeling has been proposed in this paper. The sputtered layer was replaced by a set of regularly or randomly distributed conducting spheres. Increasing sputtering time was simulated by decreasing their distance or increasing the number of spheres. The regular distribution was analyzed by the dipole model taken from [10], and by the combined model, newly proposed in this work. The latter model considers both the capacity between the individual spheres for shorter sputtering times, when the structure is not homogeneous, and the resistivity of a homogeneous layer for longer sputtering times. The reflectivity from 2-D arrays of both regularly and randomly distributed spheres was checked by simulation, using the CST Microwave Studio. All curves of the sputtering time dependence of the reflection coefficient were compared with each other and with measured data.

It was verified both by the measured data taken from [8] and [9] and by our models that sputtering is a random process. The time of the rapid increase in reflectivity may occur at different sputtering times based on the particular sputtering process or on a random distribution of the spheres in the CST Microwave Studio model. It was found on the basis of 162 CST Microwave Studio simulations that the rapid increase most probably falls at approx. $27.81 \mathrm{~s}$, with a standard deviation of $7.33 \mathrm{~s}$ and $67 \%$ of cases of the rapid increase located in the interval from (mean value minus standard deviation) to (mean value plus standard deviation), i.e., from 20.48 to 35.14 s. The measured data lay within this interval.

Our models approximate the measured data quite well in the whole range of sputtering times, recording low reflectivity for short sputtering times, when the layer is not homogeneous, a rapid increase while the conducting path is being created, and high reflectivity for long sputtering times, when the layer is homogeneous. Regular distribution of spheres results in analytic formulas for the reflection coefficient. A random distribution of the spheres is closer to the real random sputtering process, but may be analyzed only by the EM solver, a disadvantage of which is the need for time-consuming simulations. To conclude, the analytical models with regular distribution of spheres are suitable for getting a quick first idea about the features of the sputtered layer. For a subsequent more detailed insight, including the statistical distribution of the times of rapid increase in reflectivity, the time-consuming use of the EM solver is necessary.
The conductivity of a thin layer has been discussed. The results from the thin-layer model taken from [6] were compared with measured data [9]. It was found that real conductivity of the sputtered layer is much lower than that predicted by the model given in [6], due to the porous character of the real sputtered layer. The measured conductivity was therefore used when modeling the layer.

Modeling of metal nanolayers is important when it is necessary to made a sputtered layer with defined impedance, e.g., in the domain of active packaging and electromagnetic shielding.

\section{REFERENCES}

[1] V. V. Yakovlev, "Improving quality of microwave heating by packaging-Analytical approach," in Proc. 2001 ASAE Annu. Int. Meeting, Paper 01-6149.

[2] [PLEASE PROVIDE COMPLETE PAGE SPANS AS NEEDED THROUGHOUT]s. Pellicori, "Transparent electromagnetic compatibility coatings," Coating Mater. News, vol. 13, pp. $\mathbf{x x x x x}-$, Mar. 2003.

[3] G. Bosi, "Transmission of a thin film of spherical particles on a dielectric substrate: The concept of effective medium revisited," J. Opt. Soc. Amer. B, vol. 9, pp. xxxxx-, Feb. 1992.

[4] J. Aizpurua, L. Blanco, P. Hanarp, D. S. Sutherland, M. Käll, G. W. Bryant, and F. J. García de Abajo, "Light scattering in gold nanorings," J. Quant. Spectrosc. Radiat. Transf., vol. 89, pp. 11-16, 2004.

[5] H. Bosman, Y. Y. Lau, and R. M. Gilgenbach, "Microwave absorption on a thin film," Appl. Phys. Lett., vol. 82, pp. 1353-1355, Mar. 2003.

[6] R. C. Hansen and W. T. Pawlewicz, "Effective conductivity and microwave reflectivity of thin metallic films," IEEE Trans. Microw. Theory Tech., vol. MTT-30, no. 11, pp. 2064-2066, Nov. 1982.

[7] A. A. Hirsch and S. Bazian, "Electrical conduction of thin metallic layers at high frequencies down to liquid helium temperatures," Physica, vol. 30, pp. 258-264, 1964.

[8] J. Zehentner, J. Macháč, V. Langer, M. Hudlička, V. S̆vorčik, P. Slepička, and I. Drbohlav, "Experimental exploration of gold sputtered layer behaviour at microwave frequency," EMFM 2004 Proc., vol. 1, pp. 105-108.

[9] V. S̆vorčik, J. Zehentner, V. Rybka, P. Slepička, and V. Hnatowicz, "Characterization of thin Au layers on PET: Discontinuous to continuous, homogenous layer," Appl. Phys. A, vol. 75, p. 541, 2002.

[10] S. Tretyakov, Analytical Modeling in Applied Electromagnetics. Norwood, MA: Artech House, 2000.

[11] F. Nibler, High-Frequency Circuit Engineering. London, U.K.: Inst. Elect. Eng., 1996.

[12] F. M. Erickson, The capacitance between two spheres [Online]. Available: http://www.ttc cmc.net/-fme/spheres.11-03-99.ps.gz Nov. 3, 1999

[13] CST Microwave Studio [Online]. Available: http://www.cst.com/Content/Products/MWS/Overview.aspx [Online]. Available:

Pavel Buchar (S'06) received the M.S. degree from the Faculty of Electrical Engineering, Czech Technical University in Prague (CTU FEE) in 2004. He is currently working toward the Ph.D.degree in the Department of Electromagnetic Fields, CTU FEE.

$\mathrm{He}$ is an author or coauthor of more than 12 scientific papers in journals and conference proceedings. His main areas of interest are the use of nanostructures in electronics and propagation of electromagnetic waves in periodic structures.

Jan Macháč (M'99-SM'01) received the M.S. and Dr.Sc. degrees from the Czech Technical University in Prague (CTU FEE) in 1977 and 1996, respectively, and the CSc. degree (Ph.D. equivalent) from the Czechoslovak Academy of Sciences, Prague, Czech Republic.

In 1991 he became an Associate Professor at CTU FEE. He is currently an Associate Professor at CTU FEE in Prague. He is an author or coauthor of more than 130 scientific papers in journals and conference proceedings, and several textbooks. His main research interests are investigation of planar passive elements and subsystems, antennas, and periodic structures used in millimeter-wave engineering and wave theory. 
BUCHAR et al: : MICROWAVE REFLECTIVITY FROM GOLD SPUTTERED NANOLAYER

7

Dr. Macháč was a member of the Technical Program Committee of the European Microwave Conference in 1995-1997 and Secretary of this conference in 1996. He was a member of the TPC of the 2004 URSI International Symposum of Electromagnetic Theory. He is a reviewer of IEEE MTT, Electronic Letter and IEE Proc. Microwaves, Antennas \& Propagation, and reviews papers for EuMc
$A N D E$ NAMES SPELL OW JOURNALS]

Jan Zehentner (M'92-SM'96-F'06) received the M.S. and Dr.Sc. degrees from the Czech Technical University in Prague (CTU FEE) in 1962 and 1991, respectively, and the CSc. degree (Ph.D. equivalent) at the Czechoslovak Academy of Sciences, Prague, Czech Republic, in 1972. and obtained there degree in
In 1980 he became Associate Professor at CTU FEE and, in 1994, Professor. $\mathrm{He}$ is an author or coauthor of more than 150 scientific papers in journals and conference proceedings, and several textbooks. His main research interests are planar microwave and millimeter-wave circuits, modeling of devices, and field theory.

Prof. Zehentner was a chairman of the 26th European Microwave Conference. He is a member of the member of the IEEE MTT-S IMS Technical Program Committee, member of the International Program and Steering Committee of the Electromagnetic Fields and Materials Conference, International Symposium of Signals, Systems, and Electronics, member of the IEEE MTT-S Ad Com Membership Services Committee-Region 8 Chapters Coordinator, Head of the Professional Group Microwave Technique of the Czech Electrical Society. He is a member of the Editorial Board of the IEEE TRANSACTIONS ON MICROWAVE THEORY AND TECHNIQUES, Journal of Electrical Engineering, and reviewer of Radio Science, EuMC. 